\title{
LA VISIBILIDAD DE LO COTIDIANO. DIDÁCTICA, HISTORIA Y FUENTES DOCUMENTALES PARA EL ESTUDIO DE LA VIDA UNIVERSITARIA EN LA SALAMANCA MODERNA
}

\author{
The Quotidian Visibility. Didactics, History and Documentary Sources \\ to Study University Life in Salamanca at Early Modern Age
}

\author{
Francisco Javier Rubio-Muñoz \\ kopolo@usal.es \\ Universidad de Salamanca. España \\ Fecha de recepción: 01/03/2019 \\ Fecha de aceptación: 11/06/2019
}

\begin{abstract}
Resumen: Este trabajo tiene como objetivo el empleo de las fuentes primarias como recurso didáctico en la enseñanza de la Historia. Hasta hace pocos años la importancia de los archivos históricos para la investigación teórica en el ámbito de las Humanidades había eclipsado su potencial didáctico. Una de las causas ha sido la existencia de ideas preconcebidas en el tratamiento y el uso de las fuentes documentales, si bien se han producido cambios. Actualmente, en el proceso de construcción del pasado se trasciende de los espacios y usos tradicionales de las fuentes generando, con ello, una nueva cultura histórica. Teniendo en cuenta este contexto, se propone acercar y utilizar el patrimonio histórico documental en las aulas con una finalidad didáctica. La metodología se basa en el itinerario didáctico por un archivo histórico aprovechando las múltiples opciones que ofrecen estos espacios para el aprendizaje constructivo de la Historia. El contexto educativo que promueve el aprendizaje deja de ser el aula y el centro para pasar a ocupar un espacio distinto, el archivo, a través de un contacto directo con las fuentes históricas en el que se prescinde de filtros como el libro de texto. Este hecho constituye uno de sus atractivos principales, unido al componente esencialmente práctico, un aspecto que, en el caso de la enseñanza de la Historia, resulta difícil de lograr.
\end{abstract}

Palabras clave: Archivos; didáctica; Historia; Universidad de Salamanca; vida cotidiana estudiantil; Edad Moderna. 


\begin{abstract}
This paper aims to use historical documents from archives as a resource in the teaching History. The importance of historical archives for theoretical research in Humanities has overshadowed its didactic potential until a few years ago. One of the causes has been the existence of preconceived ideas in the treatment, use and research on primary sources. Fortunately, agents involved in the construction of the past have changed, transcending space and traditional uses of historical sources and creating thereby a new historical culture. Considering this context, the approach and didactic use of historical documents heritage in the classrooms is proposed. Methodology is based on the educational itinerary through an historical archive taking advantage of multiple options for constructive learning of history offered by these spaces. The educational context that promotes learning is no longer the classroom: it occupies a different space instead, the archive. The objective is to produce direct contact with historical sources in which filters such as textbooks are dispensed with. This fact is one of its main attractions, along with the essentially practical component, an aspect that, in case of teaching history, it is generally difficult to achieve.
\end{abstract}

Keywords: Archives; Didactics; History; University of Salamanca; quotidian student life; Early Modern Age.

SUMARIO: 1. Introducción. 2. Marco teórico. 3. Fuentes para la historia de la vida cotidiana estudiantil en la Edad Moderna: el testamento. 4. ¿Cómo acercar al alumnado a los archivos? Una propuesta didáctica a través de la vida cotidiana estudiantil en la Edad Moderna. 4.1. Fase introductoria. 4.2. Propuesta de trabajo de indagación y empatía histórica. 4.3. Propuesta de itinerario didáctico. 4.4. Propuesta de análisis de fuentes: el testamento estudiantil. 4.5. Propuesta de taller paleográfico: elaboración de un testamento estudiantil. 5. Conclusiones. 6. Referencias bibliográficas.

\title{
1. INTRODUCCIÓN
}

Las dificultades que actualmente presenta una disciplina como la Historia están muy relacionadas con la falta de entendimiento de los procesos históricos, como ha demostrado la dilatada trayectoria investigadora de Fontana, Prats o Pagès, entre otros. Los alumnos suelen tener problemas para empatizar con los modos de vida del pasado, algo que se agrava con el corsé que supone el currículo escolar en relación a ciertos temas. Efectivamente, la legislación educativa sobre los contenidos poco o nada dice acerca de cuestiones tan relevantes como la vida cotidiana de las sociedades pretéritas. Son, por tanto, elementos invisibles que, sin embargo, pueden y deben ser tratados en el proceso de enseñanza y aprendizaje de la Historia.

Por otro lado, la falta de empatía del alumnado, que generalmente posee una concepción mamotrética sobre la Historia, puede ser explicada en parte por el desconocimiento de las fuentes - principalmente documentales, aunque no solo- sobre las que se construye el conocimiento histórico. Por esta razón, es indispensable poner de relieve la materia prima sobre la que la sociedad actual debe aprender del ayer para entender el hoy y comprender el mañana.

En las páginas que siguen se pretende, precisamente, abordar simultáneamente ambas cuestiones, es decir, hacer visibles temas cotidianos a través de una in- 
troducción de las fuentes documentales del pasado en las aulas. Un pasado que puede estar muy próximo al presente, algo que, por ejemplo, ocurre en centros educativos como las universidades. $Y$ es que el mundo estudiantil es inherente a la evolución de las instituciones de educación superior, un hecho que puede servir de excusa para hacer de la empatía un arma de seducción que acerque la Historia a los discentes.

No en vano, la dilatada trayectoria histórica de algunas universidades como la de Salamanca -que centrará esta propuesta-traspasa años, decenios y siglos. Concretamente ocho centurias de cambios, pero también de continuidad, entre las que se dieron periodos de florecimiento y de decadencia. Así, del Estudio Salmantino ha quedado una imagen de esplendor durante la primera parte de la Edad Moderna, momento en que se convirtió en la institución de educación superior más importante de Europa. No se puede por menos que dar a conocer, desde la didáctica de la Historia, algunos de sus entresijos.

Hasta hace pocos años la importancia de los archivos históricos para la investigación teórica en el ámbito de las Humanidades había eclipsado su potencial didáctico. Una de las causas ha sido la existencia de ideas preconcebidas en el tratamiento, el uso y la investigación de las fuentes documentales. En consecuencia, la labor en los archivos se venía asociando habitualmente a dos figuras: la del archivero, cuyo trabajo transita por la paciencia y el silencio necesarios para mantener y clasificar los documentos; y la del historiador, quien, en calidad de investigador, utiliza las fuentes escritas para entender la Historia. Aunque todavía predomina esta visión clásica, poco a poco se han ido rompiendo los esquemas. Actualmente, en el proceso de construcción del pasado se trasciende de los espacios y usos tradicionales de las fuentes generando, con ello, una nueva cultura histórica.

\section{MARCO TEÓRICO}

El diálogo entre archivos y aulas para el estudio de la Historia ha ido reforzándose a lo largo de los últimos años, pasando desde tímidos acercamientos a la consolidación de proyectos colectivos. De este modo, las primeras investigaciones sobre la enseñanza de la Historia en torno a los archivos tenían un carácter disperso al terminar el siglo $\mathrm{xx}$, si bien existían precedentes en torno a grupos de investigación surgidos a finales de los setenta. Fue el caso de Germanía-75, en la línea de la historiografía marxista, e Historia 13-16 siguiendo la New History (Prats, 1989, pp. 201-210; Sallés, 2010, pp. 5, 10), que propugnaban imitar en las aulas la metodología del historiador. Ya a finales de los noventa fueron surgiendo algunas reflexiones generales sobre el tema, tanto en el ámbito europeo (Walne, 1985), como en el marco español, como son las de Pagès y Santisteban (1994, pp. 109-165), González (1994, pp. 65-71) o Estepa (1995, pp. 55-72; 2004, pp. 33-46). 
No obstante, las investigaciones enfocadas en los usos didácticos de archivos locales españoles son más numerosas, de modo que su tratamiento se hace bien desde el punto de vista de la propia institución o bien desde algunas de sus fuentes. En esta línea pueden destacarse algunos ejemplos como el de González y Martín (1995), sobre el Archivo Municipal de La Laguna, o el de Sebastiá y Blanes (1999) y Sebastiá (2001), quienes proponen algunas actividades por medio de archivos municipales valencianos en relación con el currículo de Educación Primaria, ESO y Bachillerato. Martínez y Ponce (2003, pp. 475-488), por su parte, presentan una propuesta muy concreta basada en una única fuente para estudiar el fenómeno repoblador en Almería a finales del siglo XVI. En el caso de Fernández y González (2003, pp. 555-568), se aboga por el uso didáctico de diversa documentación procedente de varios archivos de Cuenca, estableciendo algunos modelos de trabajo. Estos modelos de trabajo se elaboran a través de ejemplos seleccionados y transcritos previamente por el profesor. Además, los autores analizan situaciones y problemas del pasado mediante casuística inquisitorial y judicial.

La consolidación de esta trayectoria tuvo su eco hace más de tres lustros, momento en el que alcanzó una mayor difusión a través de grupos consolidados. La Universidad de Barcelona alberga uno de los núcleos impulsores de una línea de investigación que articula la didáctica de las Ciencias Sociales y las fuentes de archivo; es el caso de Gemma Tribó y su equipo de Arxididàctica. Su ámbito de actuación se circunscribe principalmente a Cataluña, en donde, partiendo de trabajos previos como el de Pagès y Santisteban (1994) han explorado las posibilidades de colaboración entre universidad, archivos y centros escolares, con notables resultados. Así, las Jornades Educació-Arxius del Instituto de Ciencias de la Educación de la Universidad de Barcelona son fruto de esta vía de trabajo, cuya síntesis se encuentra en algunas publicaciones como las de Tribó $(2002,2006)$. Algunos de los caminos emprendidos inciden, entre otros aspectos, en las posibilidades de la investigación sobre Historia local en las aulas dentro del paradigma humanistaecológico, el currículum escolar y los archivos o la potencialidad didáctica de las fuentes (Tribó, 2005, pp. 67-82, 107-124, 149-170). En esta línea Serrat (2002, pp. 27-36) consideraba los archivos como entornos de aprendizaje destacando algunas posibilidades de trabajo.

La madurez del alumnado es un punto clave para una intervención didáctica más eficaz, de modo que los niveles de bachillerato y universidad están centrando las últimas propuestas. Por citar algunas experiencias recientes, Reina (2009) explora la Historia de España en Bachillerato, el mismo sector del alumnado que centra la experiencia de Prieto, Gómez y Miralles (2013, pp. 1-14), aunque, en este caso, utilizan como fuentes libros parroquiales del siglo XIX. Moreno (2016, pp. 64-68) tomó como fuente el Catastro de Ensenada en el grado de Magisterio en Educación Primaria, mientras García-Morís (2016, pp. 71-85) enfocaba el trabajo en las aulas con documentación demográfica de la Edad Moderna. 
Hay que señalar que, paralelamente a esta eclosión de trabajos, también se ha generado un pensamiento reflexivo sobre las opciones y limitaciones del uso de las fuentes documentales y los archivos en la educación. Al analizar las propuestas didácticas han surgido nuevas cuestiones acerca del papel de archiveros, profesores y escolares (Vicente y Estepa, 2012, pp. 2002-2017), o las posibilidades con el tratamiento digital de la documentación histórica, como señalaba Cerdá (2010, pp. 8597). Por lo tanto, el empleo de fuentes documentales en la enseñanza de la Historia no es un tema inexplorado; así lo demuestran los paulatinos progresos en las relaciones entre las instituciones archivísticas y educativas. No obstante, todavía dista de ser un tema recurrente por el docente, debido, en gran parte, a la necesidad de conocimientos bastante especializados, disponibilidad y existencia de archivos en el entorno escolar, etc.

Santacana (2002, pp. 7-88) mencionaba acertadamente que los tres grandes problemas que ofrece el alumnado a la hora de comprender la Historia son: el uso de la Historia como justificante ideológico; el sentimiento de que la Historia les es ajena y poco útil, y la concepción de que se trata de un corpus teórico que debe aprender por imposición del profesor, asumiendo la identificación de aprender con memorizar. En relación con estas dificultades, la propuesta que se presenta tiene como objetivo principal acercar a los alumnos al pasado de una forma diferente a la habitual, dando lugar a un aprendizaje significativo. Precisamente Fernández y González (2003, p. 555) inciden sobre esta idea que relaciona los archivos con la enseñanza de la Historia, ya que surge un interés por el alumnado que permite la reflexión, el enunciado de hipótesis y la creación de una conciencia crítica y autónoma que, en suma, sienta las bases del conocimiento del pasado.

La utilización en el aula de fuentes primarias, siguiendo a Pagès y Santisteban (2010, p. 297), contribuye al conocimiento de la Historia superando la estructura de los libros de texto, además de que el estudiante, añaden Prats y Santacana (2011), es protagonista en la fabricación del conocimiento histórico. Así pues, la didáctica de la Historia pasa por acercar el estudio de los tiempos pretéritos a los alumnos, de tal modo que se hace necesario transmitir una idea dinámica sobre la forma en que el pasado ha llegado hasta nosotros.

En definitiva, esta propuesta tiene como principal objetivo el empleo de las fuentes documentales como recurso didáctico en la enseñanza de la Historia. Se pretende que los alumnos comprendan la importancia de las fuentes y los métodos de investigación que utilizan los historiadores, convirtiéndose en la excusa para que se acerquen a una parcela del pasado que queda invisibilizado; en este caso, la vida cotidiana estudiantil en la Universidad de Salamanca durante su periodo clásico o de esplendor (la Edad Moderna). Teniendo en cuenta este contexto, se propone abordar el tratamiento didáctico del patrimonio histórico documental relacionado con la Universidad de Salamanca a través de una fuente que, paradójicamente, no se encuentra entre los muros universitarios. 


\section{FUENTES PARA LA HISTORIA DE LA VIDA COTIDIANA ESTUDIANTIL EN LA EDAD MODERNA: EL TESTAMENTO}

La Universidad de Salamanca, fundada por Alfonso IX en 1218, fue uno de los centros educativos superiores más notables de la Monarquía Hispánica durante gran parte de la Edad Moderna. En este sentido, los estudios jurídicos salmantinos abastecieron de letrados al aparato burocrático del Estado, a la vez que suponía una forma de ascenso social: la llamada promoción por las letras (Pelorson, 2008; Rodríguez-San Pedro, 1986). Del mismo modo, la importancia del colectivo discente para la ciudad de Salamanca y su Universidad queda ilustrada en el hecho de que existían, en la segunda mitad del siglo XVI, más de 7000 estudiantes en una ciudad de unos 24000 habitantes, lo cual supone casi un tercio de su población; un grupo social, por cierto, muy superior al de otras grandes universidades españolas de la época, como eran Alcalá y Valladolid, con 3200 y 1300 matriculados respectivamente hacia 1580 (Kagan, 1986).

En cuanto a la vida cotidiana estudiantil durante el periodo moderno, existen trabajos sobre la sociología histórica de los escolares que hoy en día constituyen una línea de investigación abierta, los cuales se centran en las características del estudiantado, sus modos de vida (Rodríguez-San Pedro y Polo, 2001; Torremocha, 1998, 2012; Polo, 1991) o el análisis de las naciones o grupos de estudiantes según su procedencia geográfica (Rodríguez-San Pedro, 2001; Rubio-Muñoz, 2014) entre otros temas. También son novedosos algunos enfoques como el acercamiento a la vida cotidiana estudiantil a través de sus actitudes ante la vida y la muerte en la Edad Moderna, aspectos que se pueden abordar a partir de las fuentes notariales, es decir, la documentación generada por los escribanos (Rubio-Muñoz, 2016).

En el caso salmantino, la producción documental de los escribanos o notarios públicos de la Edad Moderna se conserva el Archivo Histórico Provincial de Salamanca. Esta institución iniciaba su andadura en el Palacio de Anaya de Salamanca allá por 1931. Tras los años de la Guerra Civil, en el que el archivo queda en un estado lamentable, y los años 40 del siglo xx, cuando sufre un incendio, es trasladado al edificio de las Escuelas Menores, perteneciente a la Universidad de Salamanca. Tampoco tendría aquí el mejor de los destinos, de modo que, a medida que el archivo fue recogiendo documentos de variada índole según la legislación estatal y, por tanto, el volumen de fondos era cada vez mayor, estos se fueron repartiendo por varios edificios. Tras varias décadas de avatares y cambios de destino, se crea un edificio ex profeso para albergar la documentación en 1992, si bien no se pondría en marcha hasta pasados seis años en los que hubo que acondicionar sus instalaciones. Actualmente destaca el servicio de difusión cultural y educativa del archivo, que organiza visitas guiadas y actividades pedagógicas en colaboración con centros e instituciones culturales. Además, las instalaciones del archivo cuentan, aparte de los espacios dedicados al depósito y los despachos del personal, con sala de investi- 
gadores, aula didáctica, sala de exposiciones y conferencias, laboratorio de restauración, espacios de reprografía...

Hay que mencionar que el fondo más voluminoso del Archivo Histórico Provincial de Salamanca es la documentación procedente de la Delegación de Hacienda (Catastro de Ensenada, Contaduría, etc.), si bien los protocolos notariales, tanto por volumen y antigüedad como por ser los más consultados, constituyen uno de los conjuntos más importantes. Este tipo de documentación es susceptible de ser utilizada como recurso didáctico de tal manera que los alumnos puedan comprender los modos de vida de personas comunes y semejantes a ellos que existieron más de cuatro siglos atrás.

Dentro de la documentación notarial, el testamento, en tanto que es un documento redactado en primera persona, ofrece una visión muy atractiva para el estudio retrospectivo de la vida cotidiana no solo del individuo que lo otorga, sino también de los seres humanos que le rodearon. Además, es válido para entender el imaginario acerca de la muerte y, en suma, del contexto de individuos casi anónimos que pasan de puntillas por la Historia. Existen varios tipos de testamento, siendo lo frecuente entre los estudiantes los testamentos abiertos o nuncupativos, a los que se puede añadir un codicilo o documento que modifica alguna de las mandas testamentarias, y menos común los testamentos cerrados o in scriptis, al cual acompaña la firma de siete testigos, y en ocasiones la petición de apertura ante las autoridades (Lorenzo, 1991). En este caso varios testigos son llamados a declarar acerca de la identidad del fallecido, dando a veces detalles de las circunstancias de la muerte, sus vínculos, etc. También son interesantes los inventarios post mortem, un documento que detalla las posesiones del difunto.

El valor de esta fuente documental para el estudio de la vida cotidiana es enorme ya que el momento de afrontar el final de la vida se convierte en una pequeña autobiografía que da cuenta de aspectos sociales, económicos y culturales:

- Las relaciones sociales se pueden clasificar en función del parentesco, el paisanaje y la amistad. Estos vínculos son interpretables en muchas ocasiones en un plano de igualdad -no faltan alusiones al compañero de estudios-, pero a veces denotan un clientelismo no solo con profesores, sino también entre los propios estudiantes; por ejemplo, aquellos estudiantes con menos recursos entrasen a servir a los que eran más acaudalados (Rodríguez-San Pedro y Polo, 2001).

- En el plano económico es posible investigar en el nivel de vida de los estudiantes; dónde vivían y con quién; qué bienes poseían; qué deudas tenían; a quién prestaban, etc.

- A nivel cultural, el hecho de que los testamentos sean testimonios personales invita a indagar en la intrahistoria de los sentimientos, temores, vínculos ocultos, religiosidad,... así como la formación cultural a través de sus bibliotecas. 


\section{4. ¿CÓMO ACERCAR AL ALUMNADO A LOS ARCHIVOS? UNA PROPUESTA DIDÁCTICA A TRAVÉS DE LA VIDA COTIDIANA ESTUDIANTIL EN LA EDAD MODERNA}

La variedad de temas que pueden tratarse a través de los testamentos hace que, a priori, resulten sugerentes para llevarlos al aula. Ahora bien, el tratamiento didáctico de esta fuente implica utilizar una metodología en la que el alumno consiga un aprendizaje significativo a través de la empatía con los modos de vida del pasado. En este sentido, el docente es consciente de que el conocimiento histórico no resulta, con bastante frecuencia, un elemento atractivo para los alumnos; razón de más para que, como educadores, se empleen metodologías diversas que puedan captar su interés. La Historia es un conocimiento en construcción, por tanto, su acercamiento en las aulas debe realizarse desde estrategias que incluyan la indagación como parte de la metodología de los historiadores, superando el carácter erudito o curioso para considerarse como ciencia social (Prats y Santacana, 2011, p. 18).

Una de las metodologías más efectivas a la hora de enseñar Historia es la interactiva, dentro de la cual el método de indagación dota a los alumnos de estrategias individuales encaminadas a producir un aprendizaje por descubrimiento; sería, por tanto, un método inductivo (Quinquer, 1997, pp. 100, 114). En este sentido, la investigación-acción, dentro del aprendizaje por indagación, sostiene que la construcción del conocimiento es llevada a cabo por el alumno dentro del proceso de enseñanza-aprendizaje (Elliot, 2000; Latorre, 2003). Aplicado al tratamiento didáctico de la fuente documental indicada, se darían varias etapas sucesivas:

- $\quad$ propuesta de un tema de trabajo a los estudiantes;

- planificación las tareas necesarias para lograr los objetivos;

- ejecución;

- presentación;

- $\quad$ evaluación del proceso y del resultado.

La empatía histórica juega un papel crucial en el desarrollo del proyecto, ya que la imaginación es un arma poderosa, si bien, a su vez, esta debe ser alimentada por elementos tangibles (Serrat, 2002, pp. 27-29). En este sentido, la empatía histórica puede trabajarse a través de los archivos y las fuentes documentales dado que estos se configuran como entornos de aprendizaje muy útiles para tratar diferentes contenidos conceptuales y procedimentales que normalmente quedan invisibilizados en la enseñanza de la Historia. Se trata, en fin, de trasladar al alumno al pasado por medio de una especie de viaje en el tiempo que culmina con la elaboración de un documento primario. 
Para lograr que los alumnos empaticen es indispensable que se trasladen al lugar donde se alojan las fuentes históricas. Por esta razón, el itinerario didáctico por el archivo puede incluirse aprovechando las múltiples opciones que ofrecen estos espacios para el aprendizaje constructivo de la Historia. El contexto educativo que promueve el aprendizaje deja de ser el aula para pasar a ocupar un espacio distinto, el archivo, a través de un contacto directo con las fuentes históricas en el que se prescinde de filtros como el libro de texto. Este hecho constituye uno de sus atractivos principales, unido al componente esencialmente práctico, un aspecto que, en el caso de la enseñanza de la Historia, resulta difícil de lograr. Así, el hecho de visitar un archivo histórico permite enriquecer y ampliar el léxico, al mismo tiempo que los alumnos pueden interactuar con los propios fondos documentales, utilizando algunas de las metodologías del historiador y convirtiéndose en investigadores por un día. Como se ha mencionado, con ello se desarrolla la empatía, de tal modo que, tras una experiencia de investigación fuera del aula, pueden comprender con mayor profundidad los fenómenos históricos.

Por otro lado, se trabajan casi todas las competencias a la hora de tratar las fuentes históricas y los archivos en clase, sobre todo la competencia de conciencia y expresiones culturales y las competencias sociales y cívicas, así como la competencia de aprender a aprender. El resto de las competencias se tratan de una forma indirecta: la competencia matemática, por ejemplo, a la hora de realizar cálculos sencillos con la cronología; la competencia digital a través del uso de recursos y fuentes de información volcadas en la red y la competencia del sentido de la iniciativa y el espíritu emprendedor, mediante el protagonismo que poseen los estudiantes, de cuya iniciativa personal depende el planteamiento y la resolución de problemas. Además, comprueban la existencia de actividades profesionales relacionadas con el conocimiento del pasado.

Hay que añadir que en los métodos interactivos el profesor asume un rol de guía que proporciona recursos y organiza el proceso; de ahí que sea necesaria cierta formación específica en materias como paleografía o diplomática que se suma al conocimiento disciplinar adquirido a lo largo del tiempo. Y es que la experiencia con documentos primarios requiere un diseño previo y cuidadoso que debe ser consensuado con el personal del archivo. No se trata de saturar a los alumnos con una batería de descripciones teóricas, sino contextualizar la actividad y tener la capacidad para resolver posibles dudas. En este ámbito de actuación, el contacto directo con los documentos -ver, tocar, oler la Historia- es muy recomendable, aunque no siempre posible. Por esta razón los recursos archivísticos en red disponibles hoy día complementan (y a veces sustituyen) el formato físico de las fuentes, en una «armonización entre tradición y modernidad» (Tribó, 2005, p. 26). 


\subsection{Fase introductoria}

La introducción de fuentes documentales en el aula puede hacerse desde diversas perspectivas, siendo lo recomendable comenzar desde un enfoque más generalista. En él, los alumnos pueden descubrir la importancia de los documentos como elementos que generan la Historia que convencionalmente se divide en períodos. Por esta razón, sería oportuno que los jóvenes pudieran consultar directamente algunos ejemplos documentales que abarcasen desde la época medieval hasta el período contemporáneo. Después, pueden llevarse a cabo experiencias con un tipo de fuente en concreto para lograr un proceso investigador a través del método inductivo: hipótesis-análisis de fuentes-síntesis.

La propuesta puede dividirse en varias sesiones subdividas en etapas, que parten de la introducción del tema y se indaga sobre los conocimientos previos:

- Tras conocer qué saben los alumnos sobre el tema, se realiza una visita didáctica por el Archivo Histórico Provincial de Salamanca, en donde se tendrá un contacto directo con las fuentes para así conocer la función de los documentos en la Historia.

- En segundo lugar, se les propone realizar, en grupos de trabajo, un proyecto de investigación. Este tendrá como objetivo tratar un tema invisible en el currículo educativo: "La vida cotidiana estudiantil en la Universidad de Salamanca», el cual se ubicará cronológicamente en la Edad Moderna.

- Después, tendría lugar el análisis directo con fuentes seleccionadas en el archivo, por grupos de trabajo.

- En cuarto lugar, se efectuaría un taller de paleografía y confección del soporte, con el objetivo de que puedan elaborar una fuente siguiendo la estructura formal de los ejemplos documentales examinados más a fondo en el archivo.

- Finalmente, se realiza una presentación de los resultados, antes de concluir con la evaluación final de la experiencia.

De modo introductorio se debe llevar a cabo una indagación sobre los conocimientos previos, ya que saber lo que conocen los alumnos sobre las épocas en cuestión y sobre las fuentes es muy importante para enfocar el aprendizaje constructivo en las carencias que pueden tener (Coll, 1990; Gómez, 1991). Para ello, se puede comenzar con un cuestionario en el que se aborde el concepto de fuente histórica, es decir, si tienen un concepto amplio en tanto a cualquier elemento que aporte información sobre el pasado o, en cambio, tienen una idea más particular (que haya pasado un tiempo para determinar su carácter de "histórica», o si solo se relacionan con documentos escritos, por ejemplo). También es interesante conocer si los alumnos tienen alguna noción previa de la existencia de los archivos históricos, si han visitado alguno con anterioridad o, en cambio, si desconocían la existencia de estas instituciones. 
Este sondeo puede ayudar al profesor a entender el grado de conocimiento o desconocimiento acerca de los archivos, y, por consiguiente, la ausencia o presencia de visitas previas. Las posibles causas pueden vincularse a las funciones que los alumnos arrogan a un archivo, bien como un simple edificio que contiene, almacena y conserva fuentes históricas, o bien como un centro de investigación o difusión de conocimiento. Ligado a ello también puede indagarse sobre la concepción que tienen los alumnos sobre el acceso y las posibles restricciones a este tipo de lugares, es decir, si solo pueden acceder investigadores y personas acreditadas con un interés justificado o, en cambio, el acceso a los archivos es libre para cualquier ciudadano (como ocurre en el caso de los archivos públicos).

\subsection{Propuesta de trabajo de indagación y empatía histórica}

El leitmotiv de la propuesta y, por tanto, el tema del trabajo de indagación es el de la vida cotidiana estudiantil en la Universidad de Salamanca durante la Edad Moderna. El periodo elegido se justifica por dos razones. La primera, la escasez de documentos que han llegado a nosotros sobre la vida cotidiana antes de ese momento, lo cual haría imposible trabajar con fuentes primarias. $Y$ no menos importante es el hecho de que en el imaginario colectivo de las formas de vida académica quedó grabado precisamente este momento en el que la institución alcanza su mayor esplendor.

La investigación-acción en la enseñanza y el aprendizaje de la Historia concibe el hecho educativo como una labor de investigación, que en este caso se hará en grupo. Lo importante es el propio proceso en sí y la reflexión en torno a cómo se llega al conocimiento del pasado y, en definitiva, a la adquisición de una conciencia histórica. Por esta razón, para llevar a cabo la investigación por parte del alumnado, el profesor debe facilitar a cada grupo un dosier de información con diversas fuentes, sumado a otras posibles lecturas recomendadas.

Siguiendo las instrucciones del profesor, cada grupo indagará sobre el tema en cuestión mediante el trabajo en equipo, de tal manera que, acudiendo a diversas fuentes, los alumnos elaborarán una síntesis diferente de las formas de vida de los estudiantes universitarios en la Edad Moderna. Así, tras recopilar la información suficiente deben escribir un pequeño trabajo de investigación en donde se plasmen las características más importantes del mundo estudiantil universitario, con temas como la vida académica, la indumentaria, el hábitat, la comida, las relaciones sociales, el nivel económico, etc. Con este trabajo, los alumnos fabrican un corpus teórico de contenidos para partir hacia la fase práctica, al tiempo que adquieren conocimientos nuevos.

No obstante, lo que suscita un mayor interés en esta parte es que, al consultar diversas fuentes $y$, sobre todo, al realizar un ejercicio de reflexión que supone confrontar información procedente de diversos orígenes y formatos, cada alumno y cada grupo se aproxima a la labor del historiador; algo que, sin duda, deriva en un ejercicio de empatía estimulante para iniciar la siguiente fase. 


\subsection{Propuesta de itinerario didáctico}

El uso del itinerario didáctico para tratar aspectos históricos y culturales en las aulas cuenta con una larga trayectoria que ha demostrado su eficacia (Ávila, 2003). Como parte de la educación no formal, los itinerarios didácticos tienen numerosas ventajas, principalmente las derivadas del contacto directo con la realidad. Ello permite una observación que enlaza ideas previas con la adquisición de nuevos contenidos dentro de un aprendizaje significativo, además de contribuir a la formación de un sentido crítico mediante una interacción del alumno con el contexto histórico.

Lo que se trata de esbozar aquí es la importancia de que los alumnos entiendan que existen otros espacios de aprendizaje más allá del aula. Para el conocimiento del pasado, los archivos son centros vivos en los que continuamente los investigadores obtienen la materia prima con la que se fabrica la Historia, mientras que los miembros del archivo se encargan de custodiar, ordenar, preservar y hacer accesible la documentación guardada. Es, por tanto, un lugar donde existe un trabajo multidisciplinar en el que la colaboración y la interacción entre diferentes agentes ofrece una metodología muy útil y extrapolable a las aulas. Por lo tanto, una visita didáctica al archivo requiere de un esfuerzo previo de planificación con el personal de la institución para que no se convierta en un mero paseo.

Dicho esto, el itinerario didáctico puede dividirse en varias paradas:

- Una primera parte a modo de introducción, de carácter más teórico y expositivo acerca de las distintas áreas (públicas, técnicas y de depósito), la historia del archivo y los documentos que alberga.

- Un segundo momento en el que se visitan los espacios destinados al depósito documental según diversas salas dedicadas a un tipo determinado de fuente. En cada una de estas áreas los alumnos pueden observar los documentos y su forma de agrupación (legajos) destacando los protocolos notariales, la cartografía, planos y documentos con formato especial. Además, se puede ofrecer la consulta de los documentos más antiguos, siempre que sea posible.

- A continuación, puede incluirse una parte más técnica: tareas de restauración de documentos, laboratorios de análisis y catalogación o el espacio de digitalización de los fondos.

- Por último, la visita a la sala de investigación en la cual los alumnos se informan del protocolo de acceso a las fuentes documentales: fichas, consulta de inventarios y documentos, etc. Si es posible, sería muy recomendable que los alumnos pudieran manipular una selección de documentos, siempre con la supervisión del profesor y del personal del archivo.

La documentación que puede ser mostrada en la visita didáctica varía lógicamente dependiendo del archivo. En el caso del Archivo Histórico Provincial de Salamanca, se puede observar documentación desde época medieval relacionada con 
la monarquía y los poderes eclesiásticos que fundamenta la Historia de las grandes instituciones; legajos con protocolos notariales de diversas épocas relacionados con la Historia socioeconómica, la vida cotidiana o las mentalidades de diferentes sectores sociales. También existen ejemplos que contribuyen a la enseñanza de la geografía: cartografía o algunos libros del Catastro de Ensenada. El objetivo es que los alumnos puedan comprobar la variedad de posibilidades ofrecidas por un archivo para investigar el pasado materializadas en documentos muy diferentes tanto en el formato, soporte, datación, origen, etc.

\subsection{Propuesta de análisis de fuentes: el testamento estudiantil}

Esta parte de la propuesta puede ser considerada como un instante clave en el que los alumnos empatizan con la labor del historiador justamente en el momento en el que se está fabricando el conocimiento histórico de forma directa. De todo el abanico documental señalado, el primer contacto con las fuentes de diversas épocas y tipos predispone al alumno a trabajar con la documentación histórica, pudiendo avanzar en un nivel de concreción mayor hacia el análisis de un documento en concreto. En este sentido, y previamente a la visita, el profesor consensua con los miembros del archivo la selección de varios ejemplos de testamentos estudiantiles que puedan ser examinados por los alumnos con detenimiento. No se trata de que transcriban el documento -tarea que debe ofrecerse por el profesor-, sino de acercarse a los tipos escriturarios de la época.

Es importante señalar que los testamentos estudiantiles no siempre se hacían en el momento previo a la muerte; también podía ser un documento que se redactaba gozando de buena salud (Rubio-Muñoz, 2016). Salir del ámbito familiar a estudiar a otra ciudad suponía no pocos riesgos en la Edad Moderna; de ahí que también se hicieran de forma preventiva. Por otro lado, los testamentos suelen tener una estructura más o menos fija, comenzando con fórmulas religiosas estereotipadas, seguidas de las disposiciones relacionadas con la salvación del alma (sepultura, entierro, misas,...) y, finalmente, un apartado para las donaciones, actos de caridad y resto de mandas a familiares, parientes o conocidos (López Benito, 1992). En ellas se podía incluir gastos, deudas, bienes, etc.

La finalidad es que los alumnos entiendan que, tanto el documento en sí (aspectos formales) como todo el proceso que conduce a su creación forman parte de unos modos de vida correspondientes a la realidad histórica de las sociedades del pasado; en este caso de estudiantes tal y como son los alumnos actuales. Para ello, analizarán cada documento construyendo una ficha que siga unas pautas básicas para su catalogación, descripción formal, soporte, contenido, fotografía, etc. Después indagarán en la información diversa que puede obtenerse de esta fuente y que hemos descrito anteriormente, clasificándola según sea un aspecto social, económico, cultural... 


\subsection{Propuesta de taller paleográfico: elaboración de un testamento estudiantil}

El segundo ejercicio de empatía trata de hacer que los alumnos se pongan en la piel de sus homónimos en el siglo Xvı para que conciban cómo era la vida cotidiana de los estudiantes en ese momento. Tras un contacto directamente con las fuentes, se ha intentado que fueran ellos mismos quienes fabricasen un documento a imitación de los testamentos analizados a fondo en el archivo.

A tal efecto, se imparte un taller de paleografía que tiene como objetivo comprender algunas de las formas escriturarias vistas tras la experiencia previa. Dicho taller se justifica para lograr efectivamente la empatía histórica: no solo es relevante el hecho de mantener un contacto directo con las fuentes, sino que los alumnos deben trasladarse al lugar del sujeto histórico -en este caso, un estudiante universitario de la Salamanca Moderna- ya que, de lo contrario, puede quedar en lo anecdótico. Para ello, el alumnado podrá experimentar por sí mismo a través de las formas, técnicas y soportes de la escritura en el periodo moderno, algo que trasciende de la teoría clásica que se refleja en los programas educativos.

Utilizando técnicas de simulación experimental, en el taller se elaborará el instrumental y materiales necesarios para que el alumno se traslade a un acto cotidiano en la Edad Moderna: redactar un documento, una tarea que normalmente realizaba el notario o escribano público. Para ello se fabrica una especie de cálamo por medio de plumas de ave a las que se incorpora en su extremo una plumilla. Con tinta china y con unos patrones se les muestran alfabetos de varias tipologías: gótica, cortesana, humanística y procesal, siendo lo más sencillo que los alumnos escriban con el tipo de letra humanística. La elaboración del soporte se realizaría por los propios alumnos utilizando para ello técnicas como la del café, ya que permite escribir encima del papel con tinta sin que haya problemas de absorción. Esta técnica consiste en sumergir los folios en una mezcla con café durante unos minutos para que este empape el soporte y le dé una tonalidad oscura, así como un aspecto acartonado. Una vez hecho este paso, se saca del recipiente y se tiende en una superficie lisa. Cuando se ha secado, el soporte está listo para ser utilizado.

Es, por tanto, el momento de aplicar todos los conocimientos adquiridos para realizar un testamento con tinta y pluma, imitando, en la medida de lo posible, la caligrafía aprendida. Las posibles dificultades de esta parte deben ser indicadas previamente a los alumnos, sobre todo aquellas ligadas con la ausencia de normas ortográficas (por ejemplo, el uso indiscriminado de letras como la b, v, h, mayúsculas, etc.). No obstante, conocer cómo se escribía en el pasado o la evolución del idioma también se relaciona con otras áreas, como la lingüística, lo cual respalda la visión interdisciplinar de esta propuesta.

En cualquier caso, se trata de que los alumnos reflejen su pasado inmediato, la familia, los bienes que acumulan, las relaciones sociales,... en el documento que están elaborando, igual que hacían sus colegas de antaño. Con ello se invita a la 
reflexión que se desprende acerca de la figura y la vida del estudiante universitario: un ser humano común y corriente que también forma parte de la Historia, a pesar de que su tratamiento en el aula habitualmente quede invisibilizado como otros tantos agentes históricos considerados como secundarios.

\section{CONCLUSIONES}

A través de esta propuesta y su investigación previa queda de manifiesto que los documentos, en tanto que son transmisores de información sobre una parcela del pasado, despliegan un componente didáctico innegable. Al integrarse en la práctica docente, los alumnos logran entender el pasado desde una perspectiva cercana, directa, al mismo tiempo que aumenta su predisposición, ya que se pasa de lo abstracto (el pensamiento histórico) a lo concreto (el documento que lo genera). En definitiva, la relevancia de las fuentes históricas se explica tanto por su contenido histórico como por el valor intrínseco del documento, ya que es un resto del pasado que debe ser conservado y accesible a la ciudadanía. Ello solo puede hacerse mediante un cambio de mentalidad en torno a la función de los archivos, en tanto que son instituciones que conservan, protegen y difunden la documentación de nuestro pasado, pero también centros vivos (como la propia Historia) que participan en la educación de la sociedad.

Lo que aquí se sugiere es un trabajo de empatía doble: por un lado, ponerse en la piel del historiador; por otro, con el sujeto histórico en cuestión (el estudiante universitario del siglo xVI). En el caso de los ejercicios de indagación del alumnado se ha mencionado la idoneidad de ofrecer la información en bruto sobre la que deberían trabajar por dos razones: primeramente, los temas de vida cotidiana, en tanto que son casi invisibles en el currículo, generan complicaciones a la hora de la búsqueda de información. Y, en segundo lugar, los estudiantes actuales suelen presentar problemas de filtro al examinar contenidos, sobre todo en Internet. Es en este momento en el que el profesorado, como conocedor de las necesidades del alumnado y de su contexto de enseñanza, debe establecer unos objetivos y unos contenidos adecuados, así como una metodología que favorezca el aprendizaje y una evaluación en consonancia con la dinámica del aula.

A pesar de considerar que las fuentes documentales primarias constituyen un interesante recurso didáctico, es cierto que hay limitaciones: la especialización en el tema y el reto que supone llevar a los alumnos a un archivo son motivos que a menudo impiden su realización. Además, como indican Prats y Santacana (2011), se deben adaptar los documentos al nivel del alumnado, amén de otras dificultades a las que los docentes pueden enfrentarse: la necesidad del trabajo didáctico previo de documentación para trabajar las fuentes; el lenguaje (castellano antiguo) que resulta difícil no solo para los alumnos, sino también para el profesorado, u otros 
problemas ligados a que en muchas ocasiones se impide la manipulación de las fuentes por parte del alumnado (Serrat, 2002, pp. 27-28).

No obstante, la comprensión del pasado a través de las fuentes archivísticas aporta a los alumnos herramientas para afrontar, desde una perspectiva social y crítica, los retos a los que se tendrán que enfrentar. $Y$ en el caso del profesorado, también es importante que adquieran la capacidad para reflexionar sobre qué se enseña y cómo se enseña en relación al pasado; solo así el docente podrá intervenir verdaderamente en el futuro de la sociedad.

\section{REFERENCIAS BIBLIOGRÁFICAS}

Ávila, R. M. (2003). La función de los itinerarios en la enseñanza y el aprendizaje del patrimonio histórico-artístico. Íber. Didáctica de las Ciencias Sociales, Geografía e Historia, 36, pp. 36-45.

Cerdá, J. (2010). Los archivos, un lugar para descubrir. Experiencias de dinamización cultural. En J. González (coord.), III Jornadas "Archivando»: La difusión en los archivos. Actas de las Jornadas (pp. 85-97). León: Fundación Sierra Pambey.

Coll, C. (1990). Constructivismo y educación. La concepción constructivista de la enseñanza y el aprendizaje. En C. Coll, A. Marchesi y J. Palacios (comps.), Desarrollo psicológico y educación (vol. 2, pp. 157-188). Madrid: Alianza.

Elliott, J. (2000). El cambio educativo desde la investigación-acción. Madrid: Morata.

Estepa, J. (1995). El archivo en la enseñanza de la Historia. Revista TRIA, 2, pp. 5572.

Estepa, J. (2004). El patrimonio documental y los archivos como recursos en la enseñanza de las Ciencias Sociales. En R. Rey (coord.), Aprender y enseñar con el archivo: séptimas jornadas archivísticas (pp. 33-46). Huelva: Diputación Provincial.

Fernández, A. y González, F. (2003). Uso didáctico del legado histórico conservado en los archivos. En E. Ballesteros (et al.) (coords.), El Patrimonio y la didáctica de las Ciencias Sociales, (pp. 555-568). Cuenca: Asociación de Profesores de Didáctica de las Ciencias Sociales. 
García-Morís, R. (2016). Propuesta metodológica para el uso de fuentes históricas demográficas de la Edad Moderna como recurso didáctico. Revista de Didácticas Específicas, 14, pp. 71-85.

Gómez, M. A., Sanz, A. Pozo, J. I. y Limón, M. (1991). Conocimientos previos y aprendizaje escolar. Cuadernos de Pedagogía, 188, pp. 12-14.

González, F. (1994). Las fuentes documentales: utilización didáctica en la enseñanza de la historia. Aula de innovación educativa, 23, pp. 65-71.

González, L. y Martín, U. (1995). Los archivos en la enseñanza de la Historia. La Laguna: Excmo. Ayuntamiento de La Laguna-Delegación de Cultura y Patrimonio Histórico-Artístico.

Kagan, R. L. (1986). Universidad y sociedad en la España Moderna. Madrid: Tecnos.

Latorre, A. (2007). La investigación acción. Conocer y cambiar la práctica educativa. Barcelona: Graó.

López Benito, C. I. (1992). La nobleza salmantina ante la vida y la muerte (14761535). Salamanca: Diputación de Salamanca.

Lorenzo, F. J. (1991). Muerte y ritual en la Edad Moderna. El caso de Zamora (15001800). Salamanca: Ediciones Universidad de Salamanca.

Martínez, J. M. y Ponce, P. (2003). Las actividades en Ciencias Sociales. El Patrimonio documental: los libros de apeo/repartimiento como recurso didáctico. En E. Ballesteros (et al.) (coords.), El Patrimonio y la didáctica de las Ciencias Sociales (pp. 475-488). Cuenca: Asociación de Profesores de Didáctica de las Ciencias Sociales.

Moreno, R. (2016). El Catastro de Ensenada, al alcance del aula. Íber: Didáctica de las Ciencias Sociales, Geografía e Historia, 85, pp. 64-68.

Ortega, J. I. (2016). Un acercamiento al aprendizaje significativo de la historia: la utilización de la cronística de Carlos V en el aula. En F. García, C. J. Gómez y R. A. Rodríguez (coords.), La Edad Moderna en Educación Secundaria: experiencias de investigación (pp. 27-38). Murcia: Servicio de Publicaciones de la Universidad de Murcia. 
Pagès, J. y Santisteban, A. (1994). Elements per a un ensenyament renovat de les Ciències Socials: Procediments amb fonts primàries $\mathrm{i}$ aprenentatge de la Història. I Jornades de Didàctica de les Ciències Naturals i Socials al Baix Llobregat. Col. Llorenç Sans d'Estudis del Baix Llobregat (pp. 109-165). Barcelona: Publicaciones de la Abadía de Montserrat.

Pagès, J. y Santisteban, A. (2010). La enseñanza y el aprendizaje del tiempo histórico en la educación Primaria. Cadernos Cedes, 30(82), pp. 281-309.

Pelorson, J.-M. (2008). Los letrados juristas castellanos bajo Felipe III. Investigaciones sobre su puesto en la sociedad, la cultura y el Estado. Valladolid: Junta de Castilla y León.

Polo, J. L. (1991). Estudiantes manteístas salmantinos en la Universidad de Salamanca de la primera mitad del siglo XVIII. Studia Historica: Historia Moderna, 9, pp. 23-41.

Prats, J. (1989). Las experiencias didácticas como alternativas al cuestionario oficial: reflexiones críticas sobre las experiencias de «Germanía-75» e "Historia 1316». En M. Carretero, J. I. Pozo y M. Asensio (comps.), La enseñanza de las Ciencias Sociales (pp. 201-210). Madrid: Visor.

Prats, J. y Santacana, J. (2011) Trabajar con fuentes materiales en la enseñanza de la Historia. En J. Prats (coord.), Geografía e historia. Investigación, innovación y buenas prácticas (pp. 11-37). Barcelona: Graó.

Prieto, J. A., Gómez, C. J. y Miralles, P. (2013). El uso de fuentes primarias en el aula y el desarrollo del pensamiento histórico y social. Una experiencia en Bachillerato. Clío, 39, pp. 1-14.

Quinquer, D. (1997). Estrategias de Enseñanza: los métodos interactivos. En P. Benejam y J. Pagès (coords.), Enseñar y aprender Ciencias Sociales, Geografía e Historia en la Educación Secundaria (pp. 97-121). Barcelona: Horsori.

Ramírez, G. (1996). Bases teóricas para la enseñanza de la Historia en un proyecto curricular para la ESO. Valencia: ECIR.

Reina, J. A. (2009). Historia de España. Los Archivos en la Historia de Bachillerato. Aplicación didáctica. Málaga: Ediciones didácticas y pedagógicas. 
Rodríguez-San Pedro, L. E. (1986). La universidad salmantina del Barroco, período 1598-1625. Salamanca: Ediciones Universidad de Salamanca-Caja de Ahorros y Monte de Piedad de Salamanca.

Rodríguez-San Pedro, L. E. (2001). Cátedras, grupos de presión y naciones de estudiantes en la Salamanca del siglo XVII. En E. González y L. Pérez (coords.), Colegios y Universidades I. Del Antiguo Régimen al liberalismo (pp. 107-132). México: CESU-UNAM.

Rodríguez-San Pedro, L. E. y Polo, J. L. (eds.). (2001). Vida estudiantil en el Antiguo Régimen. Miscelánea Alfonso IX, 2001. Salamanca: Ediciones Universidad de Salamanca.

Rubio-Muñoz, F. J. (2014). La nación de Extremadura en la Universidad de Salamanca durante su etapa clásica. Norba. Revista de Historia, 24/2011, pp. 225-256.

Rubio-Muñoz, F. J. (2016). Solidaridad estudiantil ante la vida y la muerte en la Universidad de Salamanca del siglo XVI. Tiempos Modernos. Revista de Historia Moderna, 32(1), pp. 179-204.

Sallés N. (2011). La enseñanza de la historia a través del aprendizaje por descubrimiento: evolución del proyecto treinta años después. Enseñanza de las Ciencias Sociales, 10, pp. 3-10.

Santacana, J. (2002). La investigación en archivos: pautas y propuestas para la escuela secundaria. Íber: Didáctica de las Ciencias Sociales, Geografía e Historia, 34, pp. 7-20.

Sebastiá, R. (coord.). (2001). Fuentes documentales en las ciencias sociales. El Archivo Municipal de Sant Vicent del Raspeig. San Vicente (Alicante): Editorial Club Universitario.

Sebastiá, R. y Blanes, G. (1999). El aprovechamiento didáctico de los Archivos y su concreción en el Archivo Municipal de Alcoi. Alicante: Universidad de Alicante.

Serrat, N. (2002). Una simbiosis archivo-escuela. Íber: Didáctica de las Ciencias Sociales, Geografía e Historia, 34, pp. 27-36.

Torremocha Hernández, M. (2012). Los estudiantes universitarios en la Edad Moderna: líneas de investigación. En L. E. Rodríguez-San Pedro, y J. L. Polo (eds.), Historiografía y líneas de investigación en historia de las universidades: 
Europa mediterránea e Iberoamérica. Miscelánea Alfonso IX, 2011 (pp. 219241). Salamanca: Ediciones Universidad de Salamanca.

Torremocha Hernández, M. (1998). La vida estudiantil en el Antiguo Régimen. Madrid: Alianza Editorial.

Tribó, G. (2002). Archivos municipales y comarcales y didáctica de la historia. Íber: Didáctica de las Ciencias Sociales, Geografía e Historia, 34, pp. 46-57.

Tribó, G. (2005). Enseñar a pensar históricamente. Los archivos y las fuentes documentales en la enseñanza de la historia. Barcelona: Instituto de Ciencias de la Educación-Universidad de Barcelona-Horsori.

Tribó, G. (coord.). (2002). Didàctica amb fonts d'arxius. Barcelona: Instituto de ciencias de la Educación-Universidad de Barcelona.

Tribó, G. (coord.). (2006). Democràcia, ciutadania i arxius. Barcelona: Instituto de ciencias de la Educación-Universidad de Barcelona.

Vicente, P. y Estepa, J. (2012). Didáctica del patrimonio documental. Qué piensa el profesorado, el alumnado y el archivero. En A. Peinado (coord.), I Congreso Internacional «El patrimonio cultural y natural como motor de desarrollo: investigación e innovación» (pp. 2002-2017). Jaén: Universidad Internacional de Andalucía.

Waine, P. (recop.). (1985). La administración moderna de archivos y gestión de documentos: el prontuario Ramp. París: UNESCO. 